\title{
Review. High pressure techniques used in the paramagnetic resonances
}

\author{
A. KRUPSKA* and M. KRUPSKI \\ Institute of Molecular Physics PAS, 17 M. Smoluchowski St., 60-179 Poznan, Poland
}

\begin{abstract}
The aim of this review-paper is to overview high pressure techniques used in the magnetic resonances in laboratories. In this review, we would like to show the techniques that lead yielding high pressure applicable in the paramagnetic resonances. We would like to draw your attention to the separation of the effects resulting from the pressure and effects from the temperature, thereby separate the volume phonon contribution. Our main intention is to show the impact of high pressure on the structure of matter, in particular on its fundamental level.
\end{abstract}

Key words: high pressure techniques, EPR spectroscopy, paramagnetic resonances.

\section{Introduction}

High pressure techniques are now being used in many fields of science - physics, chemistry, geology, biology and food science. Especially the latter (high pressure bioscience and biotechnology) develops lately, while such in physics is weaker development. Pressure acts on the structure, nature, and properties of matter. High pressure reduces a distance between particles and changes the properties of many materials, which finds application in many branches of technology: in industry, food technology, geophysics, medicine, nanotechnology etc. Pressure is applied in chemistry for studies of the nature of chemical processes. Chemical reactions under pressure were described in the first half of the 20th century. Pressure has a significant impact on living organisms. Pressure acts on different levels of biological organization: from the molecular level through cellular and structural level. One can assume that the pressure has an affect on the structure of all matter on the Earth and in the Universe.

High pressure in laboratories is used for a study of the nature of matter, especially at a quantum level. This gives us an insight into the fundamental structure of matter. Pressure, specifically, changes the position of the energy levels, splits in the zero field, impacts on the hyperfine interactions, changes interactions between particles etc. Various techniques used under pressure in the laboratory allow to keep a track of the basic level of matter. Pressure phase diagrams show the nature of the specific phase transitions of matter, such as a change of state, properties of matter etc. The pressure-temperature diagrams of phase transitions are the specific phase diagrams. The phase diagram shows, in pressure-temperature space, the lines of equilibrium or phase boundaries between the three phases of solid, liquid, and gas.

In this review-paper we would like to present the specific techniques used in the paramagnetic resonances. We focus mainly on techniques developed in our laboratory. We present the photographs and schemes used for an EPR study under pressure from our laboratory, which have never been published yet. In this paper we would also like to show how we plan to solve many problems associated with working under pressure. We are convinced that our review-work, will familiarize many researchers with the techniques of paramagnetic resonances carried under pressure

\section{A short history of high pressure studies}

The beginnings of research under high pressure date back to the early 20th century. Percy Williams Bridgman is considered a father of studies under high pressure. P.W. Bridgman invented a new device, called a diamond anvil cell (DAC). This device is allowed to obtain pressures exceeding $10 \mathrm{GPa}$. P.W. Bridgman introduced the idea of an unsupported area seal which allowed to extended a pressure range in the highpressure apparatus $[1,2]$. The idea introduced by Bridgman is now being used for the construction of devices for the generation of high pressure. A diamond anvils method is currently the most widely used to produce high pressure in laboratories, especially in optical, Raman and Mössbauer spectroscopy.

In the fifties of the 20th century Tracy Hall constructed the first multi-anvil, high pressure device able to achieve pressures of $10 \mathrm{GPa}$ and temperatures above $3000 \mathrm{~K}$ [3]. In the following years there was a gradual development of the multi-anvil devices to achieve higher and higher pressures (up to $100 \mathrm{GPa}$ at high temperatures).

In 1975 the calibration of the pressure dependence of the ruby fluorescence line to $19.5 \mathrm{GPa}$ was accurately characterized with respect to the frequency and wavelength shift. It was done at $25^{\circ} \mathrm{C}$ against the compression of $\mathrm{NaCl}$ [4].

In the early $90 \mathrm{~s}$ of the 20th century the researchers were interested in the application of high hydrostatic pressure to study of proteins. For this reason they began to construct a device for measuring proteins under pressure using NMR technique.

*e-mail: krupska@ifmpan.poznan.pl 
A. Krupska and M. Krupski

\section{Techniques used for obtain high pressure in laboratories}

The unsupported area principle, introduced by P.W. Bridgman [1] is the most important principle in all high pressure techniques. The idea of unsupported area seal is illustrated in Fig. 1.

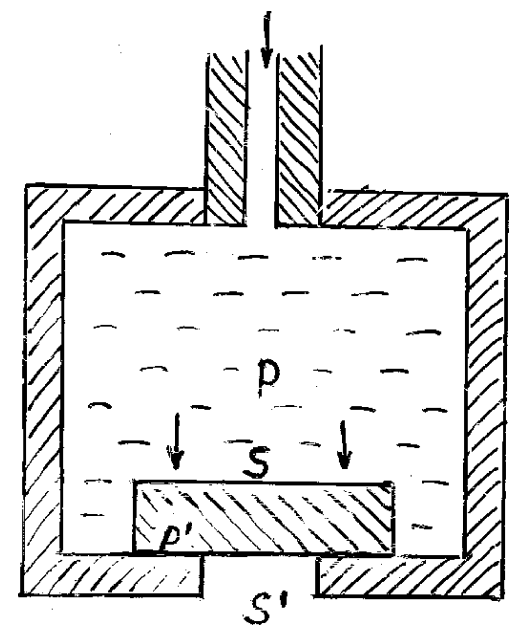

Fig. 1. Illustration of the principle of unsupported area proposed by Bridgman

$p$ denotes pressure inside and $p$ ' denotes the pressure on the gasket, $S$ - supported area, $S^{\prime}-$ unsupported area. The unsupported area $S^{\prime}$ makes the pressure on the gasket $p^{\prime}$ is larger than inside the chamber $p: p^{\prime}>p$. It follows from the following principle:

$$
p S=p^{\prime}\left(S-S^{\prime}\right)
$$

whence it follows that:

$$
p^{\prime}=p \frac{S}{S-S^{\prime}}
$$

hence: $\quad p^{\prime}>p$

We can distinguish two categories of high pressure techniques: I - gas pressure techniques, II - liquid medium techniques. The first category allows a higher generation of pressure (more than $1 \mathrm{GPa}$ ) than the second category (below the $1 \mathrm{GPa})$. The gas pressure techniques are based on a gas medium compression and typically use compressed helium gas as the pressure medium [5]. Gaseous nitrogen may also be used as the pressure medium. The liquid medium techniques are based on the use of non-polar liquid. Various liquids can be used as the pressure transmitting medium: benzoyl, light oil, petrol, petroleum ether and others. In our laboratory the petroleum ether is used as the transmitting medium.

Piston-cylinder devices are widely applied in the high pressure techniques. Figure 2 presents an example of such a device used in our laboratory.

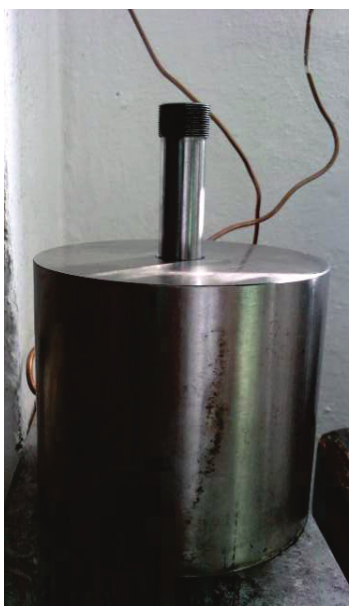

Fig. 2. Photo of one of the piston-cylinder devices used in a laboratory

In these devices both the piston and the cylinder are usually constructed of cemented tungsten carbide or wellhardened steel. Tungsten carbides have the highest compressive strength. These devices can reach pressure $3 \mathrm{GPa}$ or higher (even to $6 \mathrm{GPa}$ ). More information can be found in the book [3]. Steel used in the high-pressure pistons and cylinders should have a maximum compressive strength. Steel toughens up for obtaining hardness 62-64 HRC. There are two types of steel for the construction of high-pressure pistons: I - the tool steel type NC with a high content of C (0.95-1.1\%) and $\mathrm{Cr}(1.3-1.65 \%)$ and, II- the quick-cutting steel SW for use at elevated temperature. Steel ŁH15 classified as a first group, is the most popular and frequently used. For the constructions of high-pressure cylinders materials such as alloy steels, stainless steels and titanium alloys are used.

In order to increase the strength on the applied pressure we have applied the multilayer cylinders or process of autofrettage. The reinforcement rings are imposed on the cylinder thermally or by pressing to the cylinder reinforcement ring. In the autofrettage technique the cylinder is subjected to the high pressure which causes plastic deformation and then the pressure is released. The plasticity of the material is very important in strengthening the cylinder by the autofrettage method. Cylinders with variable mechanical sustain and chambers with hydraulic latching are also used. There are other ways of strengthening the cylinders. These include: cylinders with variable mechanical support and the chamber with a hydraulic sustain.

Diamond-anvil cells (DACs) generate very high pressure by applying force to two well-matched, gem-quality diamond anvils. The diamonds are fixed so they are perfectly aligned and parallel with each other. It relies on the operation of either a lever arm, tightening screws, or pneumatic or hydraulic pressure applied to a membrane. The operation of the diamond anvil cell relies on a simple principle:

$$
p=\frac{F}{A},
$$

where $p$ denotes pressure, $F$ - force, $A-$ area. 
Review. High pressure techniques used in the paramagnetic resonances

In DACs high pressure can be achieved by applying a moderate force $F$ on a sample with a small area $A$. Now the DACs can generate extremely static high pressure (about $300 \mathrm{GPa}$ !) Apparatus using DACs to generate pressure up to $10 \mathrm{GPa}$ at $\mathrm{X}$-band EPR spectrometer is presented in the paper [6].

The anvil cells and piston-and-cylinder are classified as the uniaxial devices. In our laboratory a special stress device has been constructed for EPR studies. The scheme and details are presented in the paper [7]. Figure 3 presents a photo of the stress device from our laboratory.

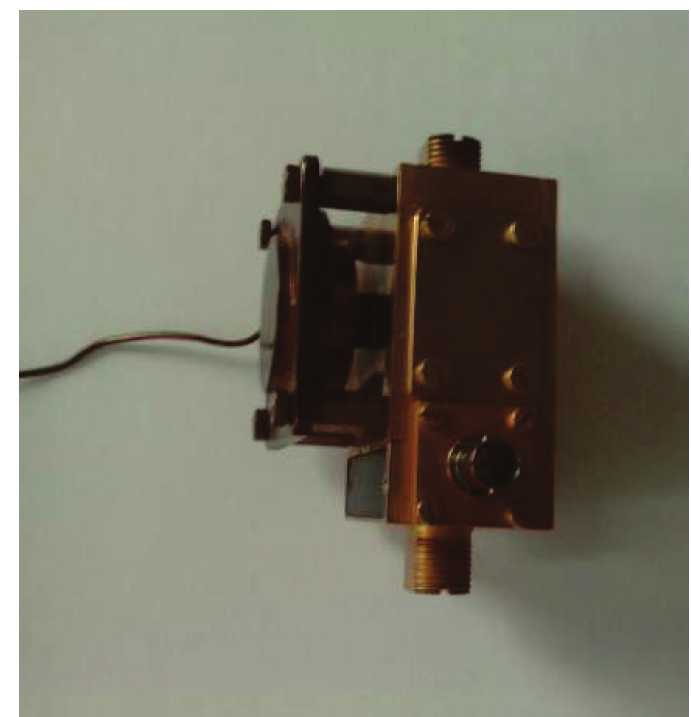

Fig. 3. A photo of the stress device from a laboratory

A hydraulic press is a device used for a production of hydrostatic pressure. The hydraulic press depends on Pascal's principle, that states that pressure exerted anywhere in a confined incompressible fluid is transmitted equally in all directions throughout the fluid such that the pressure variations (initial differences) remain the same. Figure 4 shows a diagram and working principle of a hydraulic press.

\begin{tabular}{|l|}
\hline Pressure multiplier \\
multiplication of \\
pressure
\end{tabular}

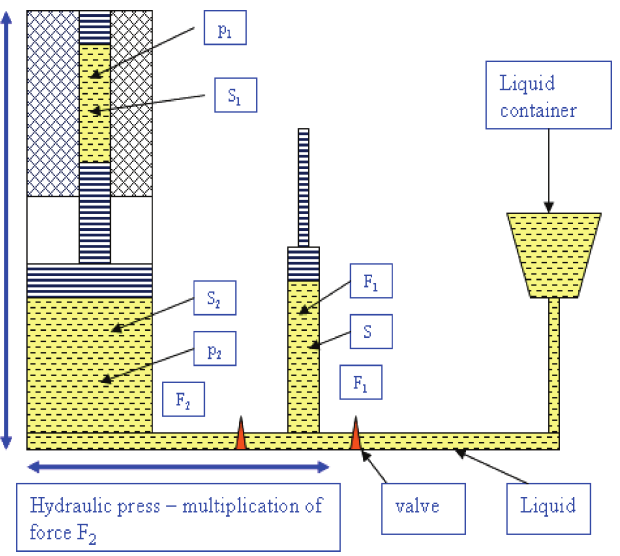

Fig. 4. Scheme and principle of operation of the hydraulic press

The hydraulic press consists of: a piston acting as a pump, with a modest mechanical force; a piston with a larger area which generates a correspondingly large mechanical force - to generate pressure and to multiply pressure, and a liquid container (oil tank). The main principle of generating pressure is as follow: 1) multiplication of force $F_{2}$ and 2) multiplication of pressure. Figure 5 shows the hydraulic press connected to the EPR spectrometer in our laboratory.

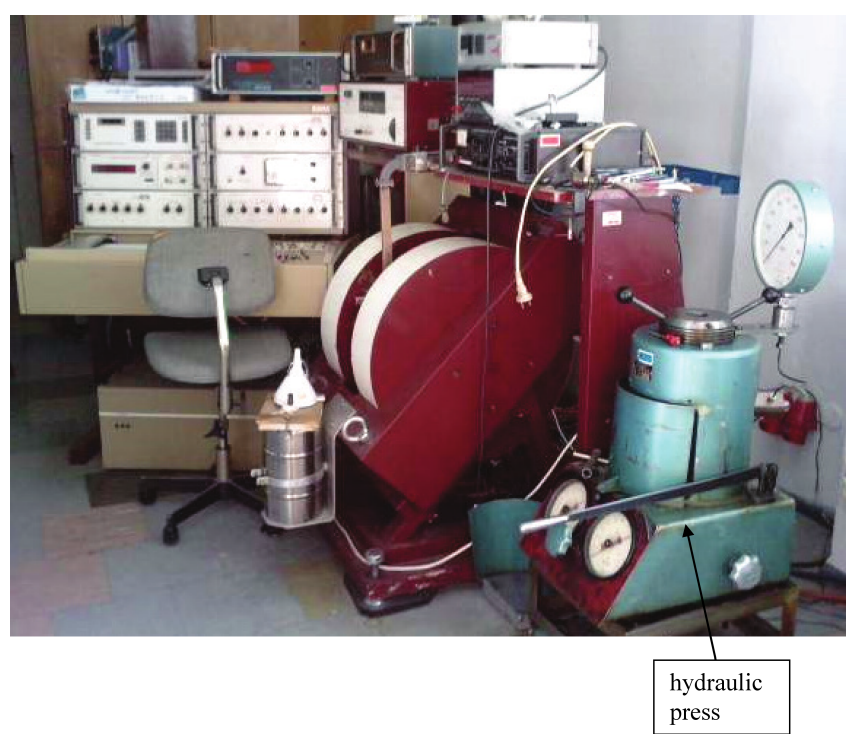

Fig. 5. Photo of the hydraulic press connected to EPR spectrometer from our laboratory

In our laboratory a special device for EPR study under high hydrostatic pressure has been constructed. Figures 6 and 7 present the photos of the high pressure EPR device used in our laboratory.

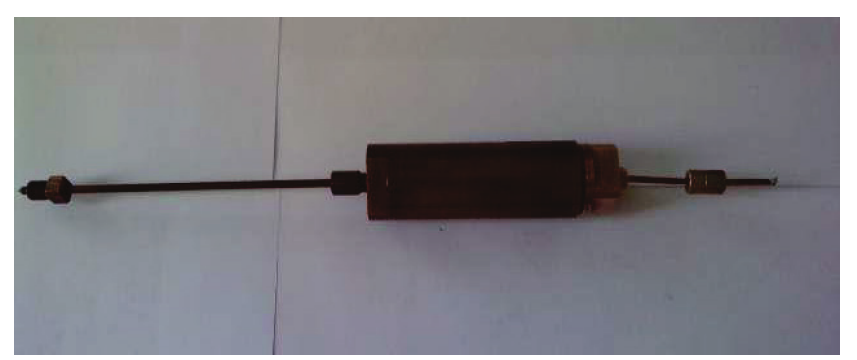

Fig. 6. Photo of high pressure device made from beryllium-bronze alloy used for EPR studies in our laboratory

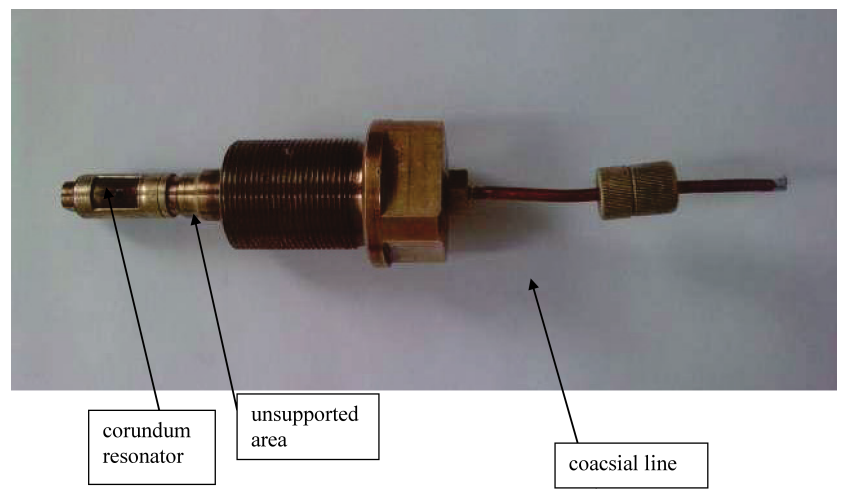

Fig. 7. Photo of a high pressure plug - the main part of high pressure EPR device (from Fig. 6) 


\section{A. Krupska and M. Krupski}

The scheme and details relating to this device are presented and described in the paper [8]. The high-pressure chamber (Fig. 6) and high pressure plug (Fig. 7) - an essential part of a high pressure EPR device - are made of a beryllium bronze alloy. All beryllium bronze elements are hardened for 3 hours at temperature of about $600 \mathrm{~K}$ after mechanical working.

A proper sealing is very important in all pressure studies. Rubber is used for O-ring. The sealing washers are made of bronze, brass, copper, aluminum, lead, indium, PCV and others. The following insulating materials are used: pyrophyllite, oxide iron, glass, quartz, mika, teflon, epoxy resins. We know that the unsupported area principle plays the most important role in the sealing. In our laboratory we use a rubber O-ring (Fig. 8 on the left) and a brass ring (Fig. 8 on the right) imposed on the high pressure plug (Fig. 7) [8]. In our laboratory this type of seal is used in both room and low temperature, up to $7 \mathrm{~K}$ for EPR study.

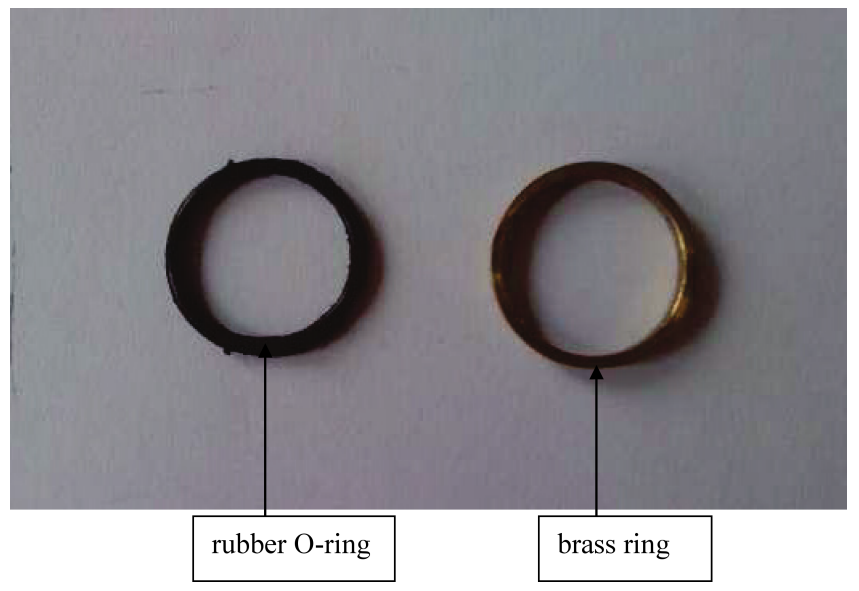

Fig. 8. Photo of high pressure seals used in our laboratory: on the left - a rubber $\mathrm{O}$ - ring, on the right $-\mathrm{a}$ brass seal

In the EPR studies under pressure corundum cylindrical resonators are usually used. Figure 9 (on the right) presents the cylindrical corundum resonator with $\mathrm{TE}_{112}$ mode used in our laboratory.

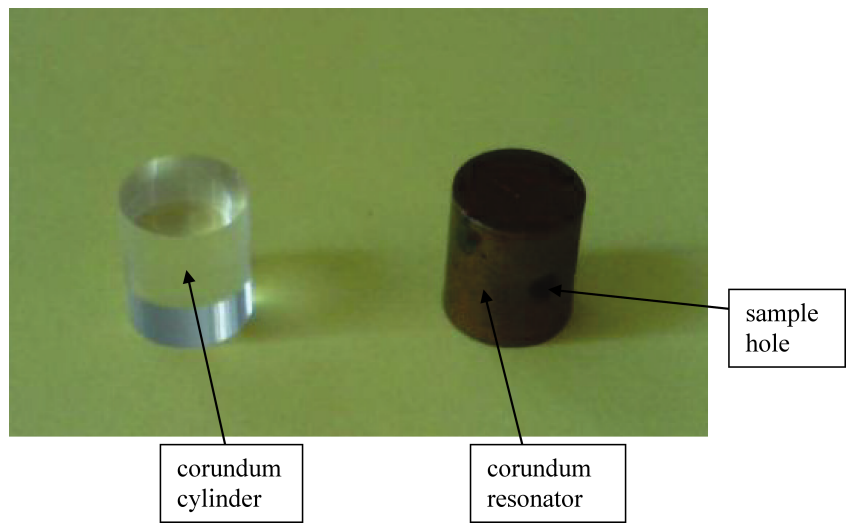

Fig. 9. Photo of the corundum ring (on the left) and next (on the right) - the resonator made from the corundum for EPR studies used in a laboratory
Its length is $10 \mathrm{~mm}$ and diameter is $2 \mathrm{~mm}$. Surfaces of our resonator are coated with a thin layer of vacuum deposited silver and then electrically reinforced by a thin layer of copper [8]. Figure 9 (on the left) presents the corundum cylinder - a fundamental material of the resonator used for high pressure EPR research in our laboratory. The scheme of lines of magnetic and electric field in our corundum resonator is presented in our paper [8].

In the NMR studies under pressure a special pressureresisting quartz cell and the protecting teflon capsule are used [9]. The maximum pressure, which is obtained in this system, is $400 \mathrm{MPa}$. This system is used for study of proteins. More information about this system is available in the book [10]. A special pressure jump unit, which sits outside the spectrometer and, through tubing, controls the pressure of a sample inside the NMR spectrometer, has been constructed in Germany [11]. A high-frequency NMR apparatus for use in pressure cell has been constructed [12].

The dielectric resonators are used for EPR studies of liquids materials and materials with the high dielectric permittivity. The details of such resonators are described in the paper [13]. In our laboratory the special dielectric resonators for EPR studies (both standard and under pressure) have been constructed. The scheme of this type of a resonator is presented in Fig. 10.

Dielectric resonators of system: wave - guide and mounted resonators

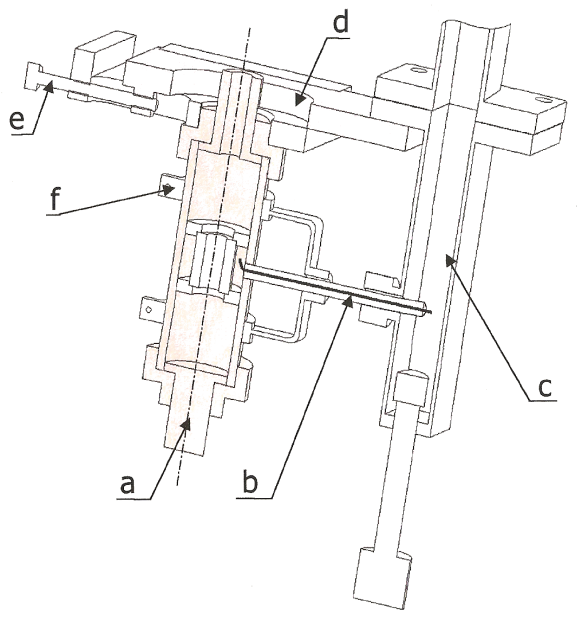
a) resonators
d) holder resonators
b) concentric cable
e) adjustment of resonator coupling
c) wave - guide
f) holder concentric cable

Fig. 10. Scheme of the dielectric resonator system made in a laboratory

Figure 11 presents the comparison of the EPR signal intensity obtained by the standard resonator and the dielectric resonator.

Figure 12 presents the photo of the dielectric resonator (on the left) and a standard resonator (on the right) used in the EPR studies in our laboratory. 
Review. High pressure techniques used in the paramagnetic resonances

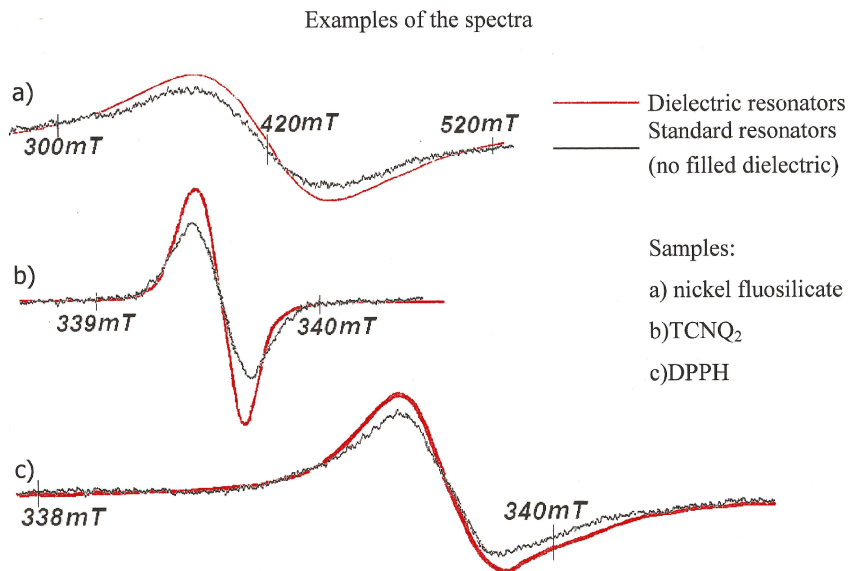

Fig. 11. Comparison the EPR spectra obtained from the dielectric resonator (red line) and the standard resonator (black line)

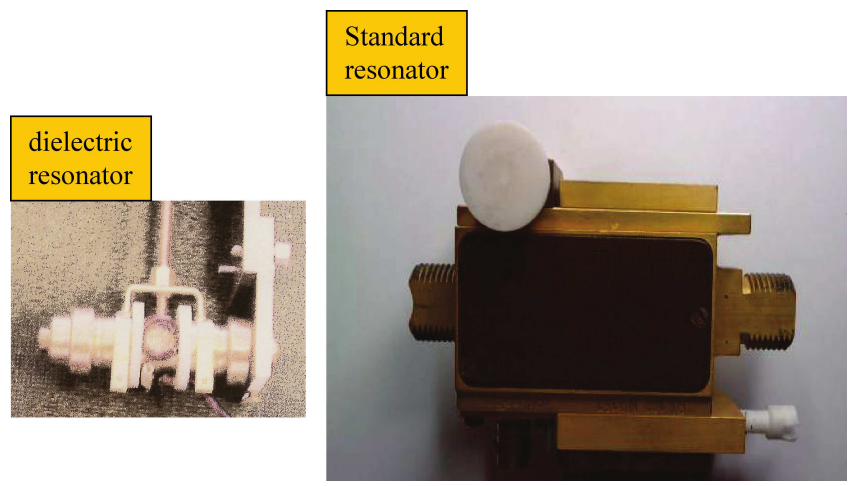

Fig. 12. Photo of the dielectric resonator made in our laboratory (on the left) and the standard resonator used for EPR studies (on the right)

\section{Separation of the effects of volume and phonon contribution}

High pressure studies as a function of temperature are often carried out in the laboratory. Defining the contribution of the temperature (phonon contribution) and the pressure (volume contribution) is necessary in all temperature studies under pressure. For this reason the separation of the effects from the pressure (volume) and effects from the temperature (phonon) is needed (Fig. 13). lows:

However, this poses some difficulties. We do this as fol-

For the regular crystals, the physical parameters $X$ such as e.g. dielectric permeability $\varepsilon$, zero field splitting $D, \mathrm{NQR}$ frequency $\nu_{N Q R}$ etc. depends on the pressure $p$ and the temperature $T: X=X(p, T)$. The pressure effect is $\left(\frac{\partial X}{\partial p}\right)_{T}$ and the temperature effect is $\left(\frac{\partial X}{\partial T}\right)_{p}$ and $\left(\frac{\partial X}{\partial T}\right)_{V}$ then:

$$
\left(\frac{\partial X}{\partial T}\right)_{p}=\left(\frac{\partial X}{\partial T}\right)_{V}-\frac{\alpha}{\beta}\left(\frac{\partial X}{\partial p}\right)_{T}^{\text {II }},
$$

where

$$
\begin{aligned}
& \left(\frac{\partial X}{\partial T}\right)_{V} \text { denotes a pure temperature effect, } \\
& \left(\frac{\partial X}{\partial p}\right)_{T} \text { denotes a pressure effect. }
\end{aligned}
$$

The first part I of Eq. (3) $\left(\frac{\partial X}{\partial T}\right)_{V}$ denotes the phonon contribution $\alpha$ :

$$
\alpha=\frac{1}{V}\left(\frac{\partial V}{\partial T}\right)_{p}
$$

The second part II of Eq. (3) $\left(\frac{\partial X}{\partial p}\right)_{T}$ denotes the volume contribution $\beta$ :

$$
\beta=-\frac{1}{V}\left(\frac{\partial V}{\partial p}\right)_{T}
$$

$V$ denotes the volume, $T$ - temperature, $p$ - pressure, $X-$ regular crystal.

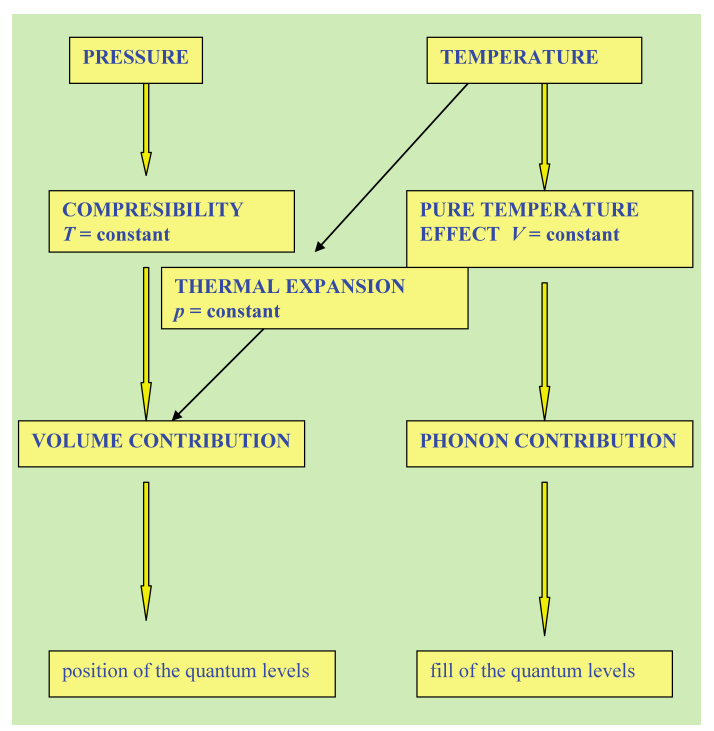

Fig. 13. Diagram showing the separation of the volume and phonon contribution in the pressure-temperatures studies

\section{Discussion - high pressure laboratory studies}

As we mentioned earlier, in laboratories high pressure is used for investigation of a structure of matter especially at the atomic, quantum level.

We can distinguish the following research techniques under high pressure used in the laboratories:

- EPR spectroscopy - would appear to be a quite good technique for a study of a matter especially in a solid subjected to high pressure. Using the EPR technique we can also carry out a study of liquids (and solutions) under pressure and even some biological structures under pressure.

- NQR spectroscopy - is used mainly for studying crystals under pressure.

- NMR studies under pressure are used for a study the solids and liquids. 
- Optical and Raman spectroscopy, Mössbauer spectroscopy, $\mathrm{X}$-Ray diffraction, neutron diffraction, AC calorimetric, dielectric studies under pressure

In this paper we have focused only on EPR, NMR and NQR studies under pressure. We have presented the essential techniques by which we can gain a high pressure in laboratories, particularly in the magnetic resonances. The table below summarizes the role of pressure in the studies of paramagnetic resonances:

\begin{tabular}{|c|c|}
\hline Technique & Role of pressure \\
\hline EPR & $\begin{array}{l}\text { - the internal changes of matter, such as a hyperfine } \\
\text { structure and fine structure, } \\
\text { - phase and spin transitions in the solids and liquids, } \\
\text { - spin-lattice relaxation, } \\
\text { - spin-spin relaxation, } \\
\text { - phase diagrams, } \\
\text { - conformational and volumetric properties in the spin } \\
\text { labeled proteins, }\end{array}$ \\
\hline NQR & $\begin{array}{l}\text { - nuclear spin-lattice relaxation, } \\
\text { - nuclear spin-spin relaxation, } \\
\text { - phase transitions in crystals, } \\
\text { - dynamical properties of crystals, }\end{array}$ \\
\hline NMR & $\begin{array}{l}\text { - nuclear spin-lattice relaxation, } \\
\text { - phase transitions in the solids and liquids } \\
\text { - study of the protein structure. }\end{array}$ \\
\hline
\end{tabular}

As evident from the table the pressure plays an important role in the study of nature of matter especially on the basic, atomic and molecular level. Pressure can generate a state of matter: physical state, phase, fill the energy levels, subtle and hyperfine interaction and others. Pressure can also affect the conformation of polymers. Studies under pressure are essential in the preparation of the phase diagrams. Paramagnetic studies under pressure, especially NMR technique, are also important in biology and biochemistry.

We know that obtaining an adequate pressure is not easy. Some problems arising from research under pressure in the paramagnetic resonances can be expressed as follows:

- It requires specialized techniques, which we are using for years in our laboratory.

- The studies under pressure require a suitable material (hardened steel or others) from which high pressure devices are built. Material from which pressure chambers are made must be well hardened. If the steel is not well-hardened pressure can destroy a pressure device. In our laboratory such situations sometimes happen. Figure 14 shows the destroyed by pressure devices (cylinders and pistons), inappropriately constructed or made of improperly hardened steel or a bad material.
- The studies under pressure require a suitable transmission medium. We use the petroleum ether in our laboratory.

- Sealing is very important in all studies under pressure. For this purpose it is necessary to perform the high precision of the unsupported area in all devices for pressure studies (see Fig. 7). In our high pressure plug (Fig. 7) there is a special place of unsupported area in the seal area. The details about this area are shown in the scheme in our paper [8].

- Studies under pressure require a good protection. A liquid method used by us is safe, but the gas method is not safe and requires a good security.

- A lot of difficulties arise in connection with separation of the effects of volume (pressure) and phonon (temperature) contribution. We deal with this problem in the way described above.

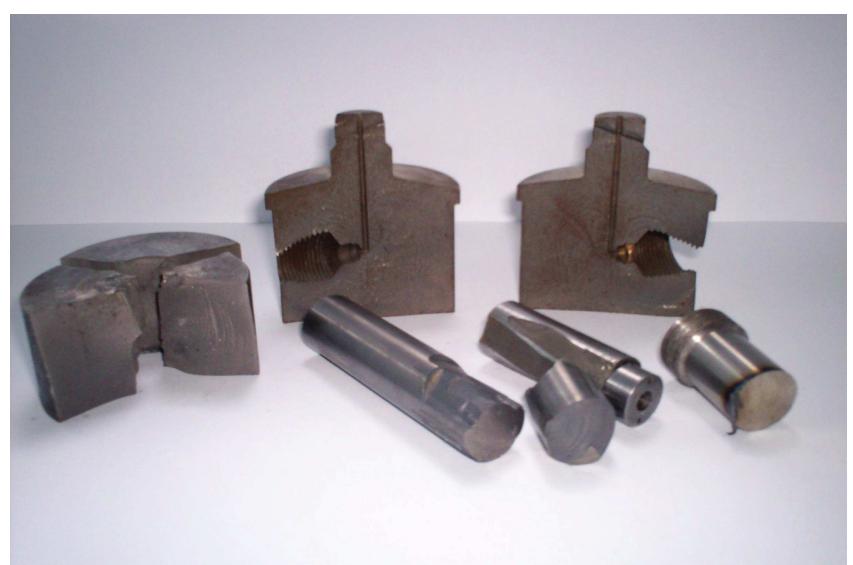

Fig. 14. Elements destroyed by the pressure devices made of bad quality material from a laboratory

Despite the many problems posed by working with the high pressures in laboratories, we think it is worth it to lead research under high pressure. This refers not only to the paramagnetic resonances presented in this paper, but also to other techniques applicable in laboratories. Electron paramagnetic resonance techniques under high pressure (up to $600 \mathrm{MPa}$ or higher) in our laboratory are well-developed, and most importantly, they are safe. In the past we also used a gas technique for EPR research, that is widely used in others laboratories in the world. However, we gave up the use of the gas technique because it is not fully safe and requires a high security level. We found some technical solutions (described in this paper) for safe and more comfortable EPR studies under high pressure.

Diamond anvil cells (DACs) allow research under higher pressure up to 25-30 GPa. The DACs are mainly used in the optical and Raman spectroscopy and Mössbauer spectroscopy in physical, chemical, and biological studies. However the DACs are rarely used in the EPR, NQR, NMR spectroscopy. However the DACs were also used in the electron paramagnetic resonance studies [6]. Recently a gasketed sapphire anvil cell (SAC) for electron spin resonance (EPR) measurements under high-hydrostatic pressures has been constructed [13]. The EPR was tested for a of 2,2-diphenyl-1-picrylhydrazyl 
radical (DPPH) positioned in the gasket of the SAC, using water as the pressure-transmitting medium [13]. Perhaps in the future the DACs will also be used in the paramagnetic resonances.

A special system for dielectric studies under pressure up to $3 \mathrm{GPa}$ has been constructed in our laboratory. The scheme and details of this system are described in the paper [14]. We hope that in the future this system can also be connected to EPR spectrometer and we will be able to perform the EPR studies under pressure up to $3000 \mathrm{MPa}(3 \mathrm{GPa})$.

\section{Conclusions}

We hope that this review allows a better understanding of the importance of the high pressure, in particular the study of matter at the quantum level using EPR, NQR and NMR techniques. We can conclude that the high pressure paramagnetic resonances techniques used in laboratories allow to study the fundamental properties of matter. Using these techniques we can study the phase transitions, spin-lattice and spin-spin relaxation, perform the phase diagrams, obtain information about the structure of intermolecular interaction and about splitting of energy levels, fine and hyperfine structure.

We hope that our review-article encourages other researchers to pursue studies under high pressure in laboratories and will be helpful to students and all who intend to begin a study under pressure with the use of paramagnetic resonances.

\section{REFERENCES}

[1] P.W. Bridgman, Physics of High Pressure, G. Bell and Sons, London, 1947.

[2] P.F McMillan, "Pressing on: the legacy of Percy W. Bridgman", Nat. Mater. 4, 715-718 (2005).
[3] H.T. Hall, Chemistry at High Temperature and Pressure in High Temperature-A Tool for the Future, Stanford Research Institute, Menlo Park, 1956.

[4] G.J. Piermarini, S. Block, J.D. Barnett, and. R.A. Forman, "Calibration of the pressure dependence of the R 1 ruby fluorescence line to $195 \mathrm{kbar}$ (19.5 GPa)", J. Applied Physics 46, 2774 (1975).

[5] I.F. Bailey, Z. Kristallogr. 218, 84 (2003)

[6] N. Sakai and J.H. Pifer, "Electron paramagnetic resonance at high pressure using a diamond anvil cell," Rev. Scl. Instrum. 56 (5), 726-731 (1985).

[7] M. Krupski, R. Kaszyński, A. Krupska, and H. Gierszal, "Uniaxial stress system for electron paramagnetic resonance studies", Rev. Sci. Instrum. 72, 4000-4001 (2001).

[8] M. Krupski, "Corrundum-filled resonator systems for highpressure and low-temperature electron paramagnetic resonance studies", Rev. Sci. Instrum. 67 (8), 2894-2898 (1996).

[9] K. Akasaka and H. Yamada, "On-line cell high-pressure nuclear magnetic resonance technique: application to protein studies," Methods Enzymol. 338, 134-158 (2001).

[10] M.W. Lassalle and K. Akasaka, "The use of high-pressure nuclear magnetic resonance to study protein folding", in: Protein Folding Protocols, ed.Y. Bay, Hardcover, London, 2006.

[11] W. Kremer, M. Arnold, C.E. Munte, R. Hartl, M. Beck Erlach, J. Koehler, A. Meier, and H.R. Kalbitzer, "Pulsed pressure perturbations, an extra dimension in NMR spectroscopy of proteins", J. Am. Chemical Society 133, 13646-13651 (2011).

[12] F. Iwase, K. Miyagawa, and K. Kanoda, "High-frequency nuclear quadrupole resonance apparatus for use in pressure cell", Review of Scientific Instruments 83, 064704 (2012).

[13] A. Sienkiewicz, B. Vileno, S. Garaj, M. Jaworski, and L. Forro, "Dielectric resonator-based resonant structure for sensitive ESR measurements at high-hydrostatic pressures", J. Magnetic Resonance 177, 278-290 (2005).

[14] J. Stankowski, S. Waplak, W. Jurga, and M. Krupski, "Sizedriven ferroelectric effects in TGS induced by high hydrostatic pressure", J. Non-Crystalline Solids 356, 1305-1309 (2010). 